\title{
Time-division multiplexing for green broadcasting
}

\author{
Pulkit Grover \\ Wireless Foundations, Department of EECS \\ University of California at Berkeley \\ Email: pulkit @ eecs.berkeley.edu
}

\author{
Anant Sahai \\ Wireless Foundations, Department of EECS \\ University of California at Berkeley \\ Email: sahai @ eecs.berkeley.edu
}

\begin{abstract}
The problem of minimizing the total (transmit and decoding) energy required for communicating over a two-receiver Gaussian broadcast channel is investigated. For achieving a specified set of rates, joint broadcast schemes (e.g. superposition coding) require smaller transmit energy per-bit than the simpler time-division multiplexing based schemes. However, for short distance communication, the energy expended in the decoding can be comparable to that required in the transmission. It is shown that in some typical short and moderate distance communication scenarios, time-division multiplexing saves on the decoding energy, thereby requiring smaller total energy than any joint broadcasting scheme for achieving the target rate and error probabilities. Further, we observe that TDM outperforms joint schemes by larger margins when the ratio of the distances of the receivers from the transmitter is closer to 1 .
\end{abstract}

\section{INTRODUCTION}

Shannon theory has been quite successful in understanding the minimum transmit power required for achieving a specified performance in many wireless network problems. In point-topoint communication, for example, waterfall curves provide a complete characterization of the minimum transmit power required to achieve a given rate and error probability. More generally, capacity theorems for average input power limited multiuser wireless network problems can be interpreted as results on the minimum required transmit power to communicate reliably at the desired rates. For some multiuser problems of practical interest, e.g. the multiple-access channel (MAC) and the Gaussian broadcast channel, the capacity region and hence the required transmit power(s) to achieve specified rates are known exactly [1, Pg. 403-407 and Pg. 427-428]. Recent results [2], [3] have succeeded in finding the required transmit power to within a constant factor for high rates for relay networks with single source and single destination as well ${ }^{1}$.

These results have found enormous applicability in optimizing the energy consumption in long distance wireless communiation networks. At shorter distances (which are of increasing interest), the processing energy is comparable to, and can even dominate the transmit energy [5], [6]. The natural problem of interest is therefore to minimize the total energy, that includes the transmit as well as the processing energy.

Processing energy has been addressed extensively in the literature on wireline networks ${ }^{2}$. However, an error free channel

\footnotetext{
${ }^{1}$ At high SNR, finding the capacity region within a constant number of bits can in most cases be interpreted as finding the required transmit power(s) within a constant factor. For a case when this is not true, see [4].

${ }^{2}$ We refer the reader to [7] for a survey of the related references.
}

or a channel that drops packets, though a good model for wired networks, is not sufficiently rich to capture important aspects (e.g. noise) of a wireless communication channel. While the information theoretic perspective on the problem has mostly been limited to the point-to-point problem [5], [7], [8] (and hence oversimplifies the network structure), the obtained results have been insightful. In [8], the authors model the transmitter as a black-box that consumes energy a constant amount of energy per unit time when the transmitter is transmitting (and hence 'on'). It consumes zero energy when the transmitter is 'off'. Unlike the results from Shannontheoretic analysis [9], the authors show that the transmissions need to be 'bursty' in that the energy minimizing rate is non-zero. A more refined model that depends on the system performance (transmit power, desired rate and target error probability) is introduced in [7]. This model accounts for the decoding energy expended at the receiver. The ensuing analysis reveals that the there is a tradeoff between the transmit and the processing energy - to minimize the total energy, transmit energy should not be studied in isolation. Further, contrary to the implication of the waterfall curve, the total energy per-bit increases unboundedly as the desired reliability increases.

To understand effects of processing energy in the design of more general networks, the authors in [10] consider the multiple-access channel (MAC). They model the processing energy at the transmitters by the black-box model that they introduced in [8]. Interestingly, the authors show that simple time-division multiple access based schemes can attain desired rates with smaller total energy than that required schemes in which the two transmitters operate simultaneously. The intuition is similar to that for the bursty strategy in the pointto-point problem: when using time-division multiplexing, one transmitter can turn itself off and save on the processing energy when the other transmitter is operating.

To investigate the impact of decoding energy in networks, it is more pertinent to consider the broadcast channel (rather than the MAC) because it is the receivers that expend the decoding energy. We provide the system model for a Gaussian broadcast problem in Section II. Of particular interest are joint broadcast schemes (e.g. superposition or dirty-paper coding) that can achieve the minimum transmit power in the limit of small error probability. In Section III-A, we provide lower bounds on the required decoding complexity (measured in number of iterations for a message passing decoder, see Section II-A) 
for a Gaussian broadcast system that uses a joint broadcast scheme. These complexity bounds are then used to provide lower bounds on the total energy consumed by joint broadcast schemes. In Section III-B we provide similar lower bounds to a time-division multiplexing (TDM) scheme that transmits to the two users in different time slots. At the cost of higher transmit energy, the TDM scheme saves on the decoding energy because the two users do not need to use the entire block to decode. In Section III-C we compare our bounds on the performance of the joint strategies and TDM, and conclude that at short to moderate distances $(\sim 1000 \mathrm{~m}$ or smaller at $3 \mathrm{GHz}$ ), TDM requires smaller total energy than joint broadcast schemes. Interestingly, it turns out that the ratio of the distances of the two receivers from the transmitter is an important factor in the design. In Section III-D, we show that allowing flexibility in the desired rate would not alter our results substantially. We conclude in Section IV.

Strictly speaking, we are comparing two lower bounds which may not be meaningful. However, as is shown in [7, Section V], such complexity lower bounds are achievable to within a constant number of iterations using regular LDPC codes. In fact, since these bounds underestimate the decoding energy, and since it is TDM that makes more efficient use of the decoding energy, we suspect that the performance of TDM vis-a-vis joint schemes would exceed the estimates provided here. This is further discussed in [11].

\section{Problem Statement}

A vector of length $m$ is denoted in bold with superscript $m$ (e.g. $\mathbf{X}^{m}$ ). A single transmitter Tx transmits to two users, user 1 and 2, across a memoryless additive white Gaussian noise channel with input $X$ and outputs $Y$ and $Z$ respectively. The information transmitted to user $i$ is a $k_{i}$ length sequence of $\operatorname{Ber}(0.5)$ iid bits denoted by $\mathbf{B}^{(\mathbf{i}){ }^{k_{i}}}, i=1,2$. The transmission is carried out in blocks of length $m$. The encoder mapping is denoted by $\psi$ that maps $\left(\mathbf{B}^{(\mathbf{1})^{k_{1}}}, \mathbf{B}^{(\mathbf{1})^{k_{1}}}\right) \rightarrow \mathbf{X}^{n}$. Thus,

$$
\begin{aligned}
& \mathbf{Y}^{m}=h_{1} \mathbf{X}^{m}+\mathbf{W}_{1}^{m} \\
& \mathbf{Z}^{m}=h_{2} \mathbf{X}^{m}+\mathbf{W}_{2}^{m},
\end{aligned}
$$

where $h_{i}$ is the distance-dependent fade coefficient, and the elements of $\mathbf{W}_{i}^{m}$ are distributed $\mathcal{N}\left(0, \sigma_{0}^{2}\right)$, are independent over $i$ and iid over time. We assume that the noises are thermal and hence $\sigma_{0}^{2}=\kappa T$ (here $\kappa$ is Boltzmann's constant, and the temperatures at the two receivers are assumed to be equal). We assume the decay in the signal power is according to the power law, that is, $h_{i}^{2}=\frac{1}{4 \pi\left(r_{i} / \lambda_{s}\right)^{2}}$, where $\lambda_{s}$ is the wavelength of the transmitted signal. Also, $r_{2} \geq r_{1}$, and hence user 1 (user 2) would also be referred to as the 'strong' ('weak') user respectively. The desired rates of communication are $R_{i}=$ $\frac{k_{i}}{m}$. Since the block length is the same for the two users, $R_{i}$ satisfy $\frac{k_{1}}{R_{1}}=\frac{k_{2}}{R_{2}}=m$. The objective is to achieve an average bit-error probability smaller than $\left\langle P_{e}^{(i)}\right\rangle_{0}$ (averaged over the channel realizations and the messages) for $i=1,2$. We will sometimes consider an AWGN test channel pair $(G, J)$ where the first user has noise variance $\sigma_{G}^{2}$, and the second user has noise variance $\sigma_{J}^{2}$. The symbol 0 is reserved for the underlying channel of noise variance $\sigma_{0}^{2}$. Under a test channel pair $(G, J)$, the average bit-error probability is denoted by $\left\langle P_{e}^{(1)}\right\rangle_{G}$ for user 1 , and by $\left\langle P_{e}^{(2)}\right\rangle_{J}$ for user 2 . We denote the average transmit power by $P$. For simplicity we assume that $m=\frac{k_{1}}{R_{1}}=\frac{k_{2}}{R_{2}}$ and hence the two messages are conveyed in the same block of length $m$. Probability of noises $\mathbf{W}_{1}^{m}$ taking values in a set $A$ under a test channel $G$ would be denoted by $\operatorname{Pr}_{G}(A)$. Similar notation is used for $\mathbf{W}_{2}^{m}$ under a test channel $J$ and for the underlying channel. The parameter $\zeta=\frac{r_{2}^{2}}{r_{1}^{2}}=\frac{h_{1}^{2}}{h_{2}^{2}}$ is of interest as explained in Section I.

\section{A. Decoding energy model}

Our focus is on the parallelism of the decoders and the energy consumed within them. The decoding energy model is borrowed from [7] which is based on the iterative decoding model [12]. We assume that each decoder is physically made of computational nodes that pass messages to each other in parallel along physical (and hence unchanging) wires. A subset of nodes are designated 'message nodes' in that each is responsible for decoding the value of a particular message bit. Another subset of nodes, called the 'observation nodes' has members that are each initialized with at most one observation of the received channel output symbols. There may be additional computational nodes to merely help in decoding. In a departure from the model in [7] [13], we assume that the observation nodes and the message nodes are disjoint. This allows simplicity in our exposition, while not altering the flavor of our results.

Each computational node is connected to at most $\alpha+1>$ 2 other nodes (an implementation constraint) with wires that allow for bidirectional communication. No other restriction is assumed on the topology of the decoder. In each iteration, each node sends messages to all its neighboring nodes. The maximum of all the neighborhood sizes (over all the message nodes) at the decoder of user $i$ at the end of $l_{i}$ iterations is denoted by $N_{i} \lesssim \alpha^{l_{i}}$. Each computational node is assumed to consume a fixed $E_{\text {node }}$ joules of energy at each iteration. We define the parameter $\gamma=\frac{E_{\text {node }} h_{1}^{2}}{\sigma_{0}^{2} \log _{2}(\alpha)}$ that captures the energy and the architecture terms relevant to our energy calculations.

\section{B. Joint broadcast and time-division multiplexing strategies}

We call a strategy a joint broadcast strategy if it requires each user to use the entire block to decode its own message. For example, superposition coding and dirty-paper coding are joint broadcast strategies. Also, we define a time-division multiplexing (TDM) strategy as one in which the signal for each user is sent at different time indices, and thus user $i$ only uses indices assigned to itself to perform the decoding. We will analyze the performance of both of these strategies. The term total energy refers to the sum of the transmit energy $E_{T}$ and the decoding energy $E_{d e c}^{(i)}$. Our objective is to minimize the total energy per-bit, that is given by

$$
E_{p e r-b i t}=\frac{E_{T}+E_{d e c}^{(1)}+E_{d e c}^{(2)}}{k_{1}+k_{2}}
$$




\section{LOWER BOUNDS ON TOTAL ENERGY}

\section{A. Lower bounds on total energy for joint broadcast schemes}

The main result of this section is a lower bound on the total energy for joint broadcast strategies defined in Section II-B. It is presented in a sequence of three theorems. Theorem 1 derives lower bounds on the bit-error probabilities for the two users under some test channels. Theorem 2 uses results of Theorem 1 to derive lower bounds on neighborhood sizes (as in [7]) for given target error probabilities. These lower bounds are eventually used to derive to lower bounds on the total energy in Theorem 3.

Theorem 1: For a test channel pair $(G, J)$ for the two-user broadcast channel of Section II, the following lower bounds hold on the error probabilities for all coding schemes that operate with average transmit power $P$ and for all $\sigma_{G}^{2} \leq \zeta \sigma_{J}^{2}$,

$$
h_{b}\left(\left\langle P_{e}^{(1)}\right\rangle_{G}\right) \geq 1-\frac{\log _{2}\left(1+\frac{h_{1}^{2} P_{u}^{\psi}}{\sigma_{G}^{2}}\right)}{2 R_{1}}=: \delta_{1}\left(P_{u}, \sigma_{G}^{2}\right)
$$

$h_{b}\left(\left\langle P_{e}^{(2)}\right\rangle_{J}\right) \geq 1-\frac{\log _{2}\left(1+\frac{h_{2}^{2}\left(P-P_{u}^{\psi}\right)}{h_{2}^{2} P_{u}^{\psi}+\sigma_{J}^{2}}\right)}{2 R_{2}}=: \delta_{2}\left(P_{u}, \sigma_{J}^{2}\right)$,

for some $0 \leq P_{u}^{\psi} \leq P$. Further, $P_{u}^{\psi}$ is dependent only on the encoding strategy $\psi$, and not on the channels $G$ and $J$.

Proof: See [11].

The derivation of Theorem 2 uses the following lemma from [7, Lemma 10].

Lemma 1: Let the underlying AWGN channel be of noise variance $\sigma_{0}^{2}$. Consider a test channel $G$ of noise variance $\sigma_{G}^{2}>$ $\sigma_{0}^{2}$. Let $A$ be a set of noise realizations $\mathbf{w}^{n}$ of length $n$ such that $\operatorname{Pr}_{G}\left(\mathbf{w}^{n} \in A\right)=\delta$. Then,

$$
\operatorname{Pr}_{0}\left(\mathbf{w}^{n} \in A\right) \geq f_{G}(n, \delta),
$$

where,

$$
\begin{aligned}
f_{G}(n, x):= & \frac{x}{2} \exp \left(-n D\left(\sigma_{G}^{2} \| \sigma_{0}^{2}\right)\right. \\
& \left.-\sqrt{n}\left(\frac{3}{2}+2 \ln \left(\frac{2}{x}\right)\right)\left(\frac{\sigma_{G}^{2}}{\sigma_{0}^{2}}-1\right)\right) .
\end{aligned}
$$

Further, $f_{G}(n, \cdot)$ is a convex- $\cup$ increasing function for any fixed $n$ and for all values of $\sigma_{G}^{2} \geq \sigma_{0}^{2}$.

Proof: See [7, Lemma 10].

Theorem 2: For AWGN broadcast channel with total average input power $P$, the following pair of equations provide lower bounds on the neighborhood sizes at the two decoders for the decoding model of Section II-A for given bit-error probabilities $\left\langle P_{e}^{(i)}\right\rangle_{0}$ at the two users.

$$
\begin{aligned}
& \left\langle P_{e}^{(1)}\right\rangle_{0} \geq f_{G}\left(N_{1}, \frac{h_{b}^{-1}\left(\delta_{1}\left(P_{u}, \sigma_{G}^{2}\right)\right)}{2}\right) \\
& \left\langle P_{e}^{(2)}\right\rangle_{0} \geq f_{J}\left(N_{2}, \frac{h_{b}^{-1}\left(\delta_{2}\left(P_{u}, \sigma_{J}^{2}\right)\right)}{2}\right)
\end{aligned}
$$

for all $\sigma_{G}^{2}, \sigma_{J}^{2}$ satisfying $\sigma_{G}^{2}<\zeta \sigma_{J}^{2}$ and for some constant $P_{u} \in[0, P]$ that depends only on the coding scheme. Here $\delta_{i}\left(\sigma^{2}\right)$ are as defined in Theorem 1.
Proof: See [11].

Turned around, these bounds provide lower bounds on the required neighborhood size $N_{i}$ for given error probabilities. Using $l_{i} \geq \frac{\log _{2}\left(N_{i}\right)}{\log _{2}(\alpha)}$, the theorem provides lower bounds on the number of iterations. Using this lower bound on the number of iterations, Theorem 3 derives lower bounds on the total energy per-bit for joint broadcast strategies.

Theorem 3 (Energy per-bit for joint strategies): The total per-bit energy required by any joint broadcast strategy for communicating at rates $R_{1}, R_{2}$ to the two users is lower bounded as follows.

$$
\begin{array}{r}
\frac{E_{\text {per-bit }} h_{1}^{2}}{\sigma_{0}^{2}} \geq \min _{P}\left\{\frac{P h_{1}^{2}}{\left(R_{1}+R_{2}\right) \sigma_{0}^{2}}+\gamma \min _{P_{u}}\left(\max _{\sigma_{G}^{2}}\{\right.\right. \\
\frac{R_{1}+1}{R_{1}+R_{2}} \log _{2}\left(N_{1}\left(P, \sigma_{G}^{2}, P_{u}\right)\right)+\frac{R_{2}+1}{R_{1}+R_{2}} \times \\
\left.\left.\left.\max _{\sigma_{J}^{2}>\frac{\sigma_{G}^{2}}{\zeta}}\left\{\log _{2}\left(N_{2}\left(P, \sigma_{J}^{2}, P_{u}\right)\right)\right\}\right\}\right)\right\},
\end{array}
$$

where the functions $N_{1}$ and $N_{2}$ are lower bounded as in Theorem 2, and the optimization is over

$$
\begin{aligned}
P \geq & \frac{1}{h_{1}^{2}}\left(2^{2 R_{1}\left(1-h_{b}\left(\left\langle P_{e}^{(1)}\right\rangle_{0}\right)\right)}-1\right) 2^{2 R_{2}\left(1-h_{b}\left(\left\langle P_{e}^{(2)}\right\rangle_{0}\right)\right)} \sigma_{0}^{2} \\
& +\frac{1}{h_{2}^{2}}\left(2^{2 R_{2}\left(1-h_{b}\left(\left\langle P_{e}^{(2)}\right\rangle_{0}\right)\right)}-1\right) \sigma_{0}^{2},
\end{aligned}
$$

and $P_{u}$ satisfying $h_{b}\left(\left\langle P_{e}^{(i)}\right\rangle_{0}\right) \geq \delta_{i}\left(\sigma_{0}^{2}\right)$ for $i=1,2$.

Proof: See [11].

\section{B. Lower bounds on total energy for TDM}

Theorem 4 (Energy per-bit for TDM): The total energy per-bit for the time-division multiplexing scheme (under the model described in Section II-A) that communicates $k_{i}$ bits to user $i$ at rate $R_{i}$ (so that $\frac{k_{1}}{R_{1}}=\frac{k_{2}}{R_{2}}$ ) is lower bounded by

$$
\begin{aligned}
& \frac{\left(R_{1}+R_{2}\right) E_{\text {per-bit }} h_{1}^{2}}{\sigma_{0}^{2}} \geq \min _{\widetilde{R}_{1}, P_{1}, P_{2}}\left\{\frac{P_{1} h_{1}^{2} R_{1}}{\sigma_{0}^{2} \widetilde{R}_{1}}+\frac{P_{2} h_{1}^{2} R_{2}}{\sigma_{0}^{2} \widetilde{R}_{2}}\right. \\
+ & \left.\gamma \frac{\left(\widetilde{R}_{1}+1\right) R_{1}}{\widetilde{R}_{1}} \log _{2}\left(N_{1}\right)+\gamma \frac{\left(\widetilde{R}_{2}+1\right) R_{2}}{\widetilde{R}_{2}} \log _{2}\left(N_{2}\right)\right\},
\end{aligned}
$$

where $\widetilde{R}_{1} \geq R_{1}$, and $\widetilde{R}_{2}$ satisfies $\frac{R_{1}}{\widetilde{R}_{1}}+\frac{R_{2}}{\widetilde{R}_{2}}=1$. Also, $h_{i}^{2} P_{i} \geq\left(2^{2 \widetilde{R}_{i}\left(1-h_{b}\left(\left\langle P_{e}^{(i)}\right\rangle_{0}\right)\right)}-1\right) \sigma_{0}^{2}$, and $N_{i}$ is lower bounded as follows

$$
\left\langle P_{e}^{(i)}\right\rangle_{0} \geq f_{i}\left(N_{i}, \frac{h_{b}^{-1}\left(\delta^{(i)}\left(\sigma_{i}^{2}\right)\right)}{2}\right),
$$

where

$$
\delta^{(i)}\left(\sigma_{i}^{2}\right)=1-\frac{1}{2 \widetilde{R}_{i}} \log _{2}\left(1+\frac{h_{i}^{2} P_{i}}{\sigma_{i}^{2}}\right),
$$

for all $\sigma_{i}^{2}$ satisfying $\delta^{(i)}\left(\sigma_{i}^{2}\right)>0$. These lower bounds can be optimized over $\sigma_{i}^{2}$.

Proof: See [11]. 


\section{Performance comparison}

In this and the following section we assume $R_{i}=R_{\text {des }}=$ $1 / 3$ (the desired rate), $k_{1}=k_{2}, E_{\text {node }}=1 \mathrm{pJ}$, temperature $T=300 \mathrm{~K}$, and an operating frequency is $3 \mathrm{GHz}$. Fig. 1 shows a comparison of the normalized total energy per-bit (given by $\frac{E_{p e r-b i t} h_{1}^{2}}{\sigma_{0}^{2}}$ ) for various values of $r_{1}$ and $\zeta=\frac{r_{2}^{2}}{r_{1}^{2}}$. For small $\zeta$, the performance gain of TDM is substantially better than any joint scheme for distances as large as 3000 $\mathrm{m}$ for the given system parameters. To understand why this must be the case, consider $\zeta=1$, the case of equal fade coefficients. It turns out that in this case, TDM also achieves the capacity of the Gaussian broadcast channel, and is hence transmit-power optimal. Intuitively, since TDM makes more efficient use of decoding energy, it should require smaller total energy for $\zeta=1$.

For larger $\zeta$, the required transmit power using joint schemes vis-a-vis that required by TDM is much smaller, and joint schemes start dominating TDM (see Fig. 2). This is particularly true at large distances, or high error probabilities, where decoding energy ceases to matter. Again, if error probability is lowered for fixed $\zeta$ (see Fig. 3), TDM outperforms joint broadcast schemes because TDM's savings in decoding energy exceed its spending on transmit energy.

Even though Fig. 1 suggests that joint schemes dominate TDM at small distances $(\sim 300 \mathrm{~m}$ or smaller at $3 \mathrm{GHz}$, depending on $\zeta$ ), we believe that this is a consequence of the looseness in our bounds. An increase in transmit power can force the lower bounds on the neighborhood sizes to 1 , thereby making the lower bound on the decoding energy zero, even though the actual decoding energy itself is non-zero.

\section{Allowing flexibility in the communication rate}

For point-to-point communication, [13] suggests that for a given target error probability, the optimal rate $R^{*}$ that minimizes the total energy per-bit can be non-zero. Consequently, it is desirable to have the operating rate $R=R^{*}$ if $R^{*}>R_{\text {des }}$.

To see if this improves the performance of the joint broadcast schemes, we allow for flexibility in $R$ subject to $R>R_{\text {des }}$. Fig. 4 shows that at very short distances, the total power is an increasing function of the rate, and hence $R^{*}=0$ (again, this happens due to the looseness of our bounds). At extremely large distances, the decoding energy is irrelevant, and $R^{*}$ approaches 0 . At moderate distances, for the system parameters in Fig. 1, 2 and 3, $R^{*}$ for joint broadcast schemes turns out to be smaller than $R_{d e s}=1 / 3$ (see Fig. 4), and hence the same plots are observed even allowing for the rate flexibility. Though there would be an impact of flexible rate at extremely low error probabilities, it would benefit both TDM and joint broadcast schemes, and thus needs further study.

\section{DISCUSSIONS AND CONCLUSIONS}

We note that the lower bounds on the joint broadcast schemes here are optimistic because they assume that user 1 can decode its own message without decoding any part of the message for user 2. In practice, for the two well known joint broadcast schemes of superposition and DPC,
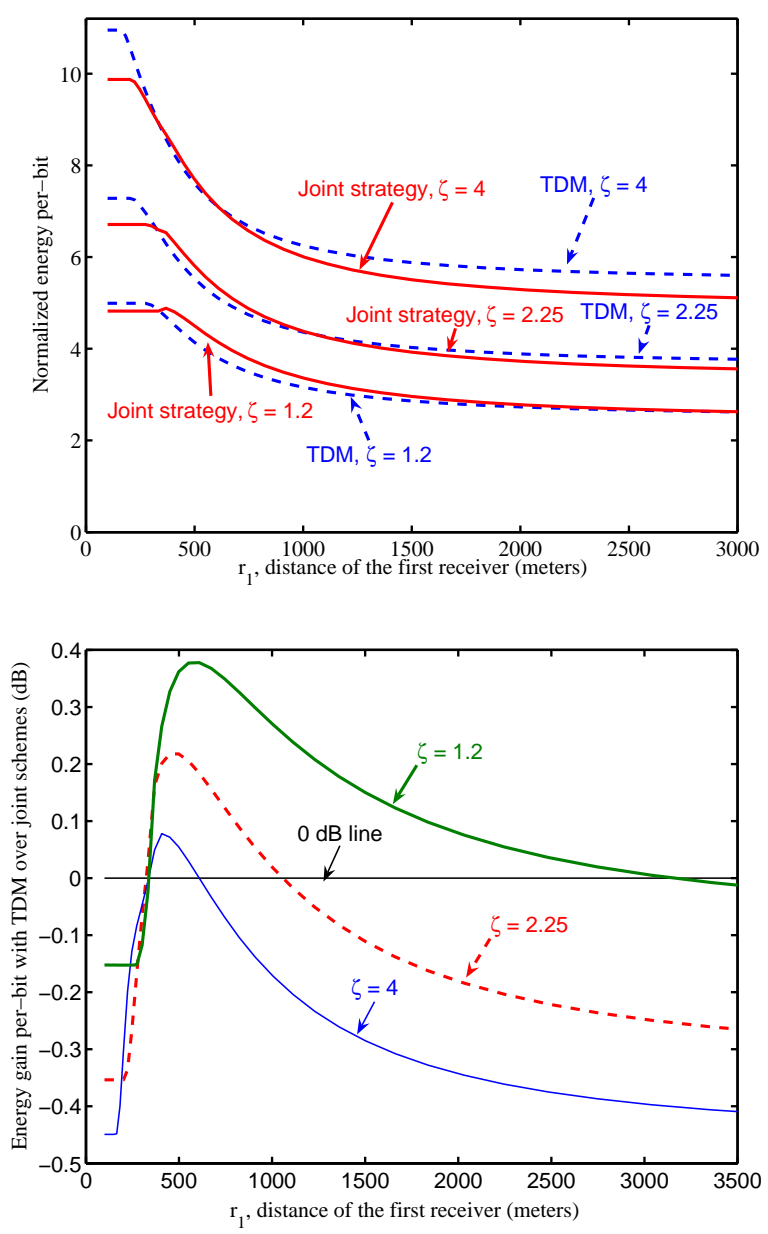

Fig. 1. The first plot shows the lower bounds on the (normalized) energy per-bit vs distance of the first user for joint broadcast schemes and TDM for different values of $\zeta=r_{2}^{2} / r_{1}^{2}$ for $\left\langle P_{e}^{(i)}\right\rangle_{0}=10^{-9}$. The total energy per-bit can be smaller for TDM for short distances and reasonably small values of $\zeta$. The second plot shows the difference in energy per-bit (in $\mathrm{dB}$ ) for TDM vs joint broadcast schemes. At moderate distances, the gap can be large for $\zeta$ close to 1 , while the advantage of TDM is small at large $\zeta$. The plots are for a transmit frequency of $3 \mathrm{GHz}$, an $E_{\text {node }}$ value of $1 \mathrm{pJ}, \sigma_{0}^{2}=\kappa T$ with $\kappa$ as Boltzmann's constant and $T=300 \mathrm{~K}$, and the y-axis shows the normalized energy $\frac{E_{p e r-b i t} h_{1}^{2}}{\sigma_{0}^{2}}$. The figure assumes $k_{1}=k_{2}$, and $R_{1}=R_{2}=1 / 3$.

user 1 decodes more bits than merely its own message bits. In superposition, user 1 decodes the entire message for user 2 . In DPC, user 2 decodes an auxiliary codeword. The number of such auxiliary codewords far exceeds the number of possible messages for user 1. Thus for either superposition or DPC, the decoding energy at the first user is higher than that assumed in Theorem 3 because the nodes dedicated to decoding these extra bits consume energy as well.

The minimum energy communication scheme for broadcast channels at short distances must have some aspect of TDM, that is, each receiver should not require the entire block for decoding. We believe that the optimal scheme would time-share between a joint scheme and TDM, thus balancing between loss in the rate in TDM and increase in the decoding 


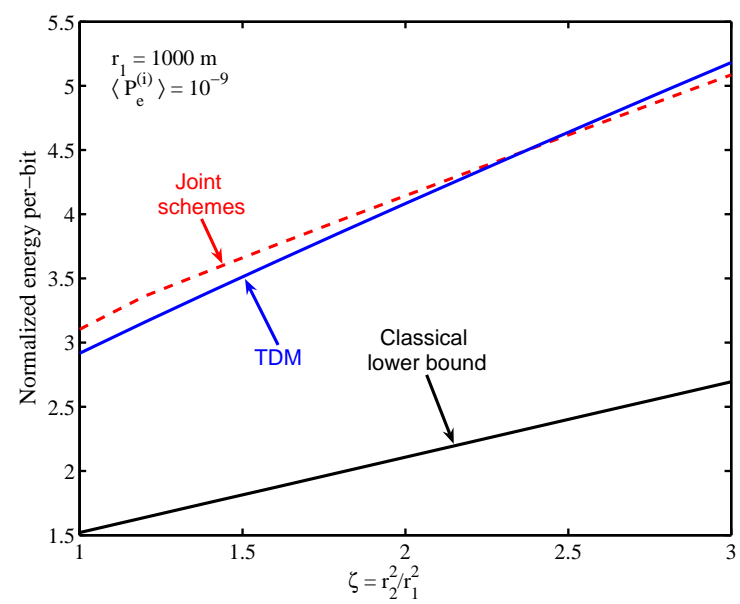

Fig. 2. The plot shows the lower bounds on the (normalized) energy per-bit vs $\left\langle P_{e}^{(i)}\right\rangle_{0}$ for joint strategies and TDM for $\left\langle P_{e}^{(i)}\right\rangle_{0}=10^{-9}$. For $\zeta$ close to 1 , TDM requires smaller energy than the joint broadcast schemes. For $\zeta$ much larger than 1, the joint broadcast schemes outperform TDM due to improved savings in transmit energy. The other parameters are the same as in Fig. 1. The classical lower bound disregards the decoding energy.

power in the joint schemes.

The connection with work of Weng et al [14] on the error exponents for broadcast channels is still under exploration.

\section{ACKNOWLEDGMENTS}

We thank Amin Gohari for useful discussions. We gratefully acknowledge the support of the National Science Foundation (CNS-403427 and CCF-729122) and Sumitomo Electric.

\section{REFERENCES}

[1] T. M. Cover and J. A. Thomas, Elements of Information Theory, 1st ed New York: Wiley, 1991.

[2] A. Avestimehr, S. Diggavi, and D. Tse, "A deterministic approach to wireless relay networks," in Proc. of the Allerton Conference on Communications, Control and Computing, October 2007.

[3] A. Avestimehr, "Wireless network information flow: A deterministic approach,” Ph.D. dissertation, UC Berkeley, Berkeley, CA, 2008.

[4] A. Lapidoth and S. M. Moser, "Capacity bounds via duality with applications to multiple-antenna systems on flat-fading channels," IEEE Trans. Inform. Theory, vol. 49, no. 10, pp. 1019-1030, Oct. 2003.

[5] S Cui, AJ Goldsmith and A Bahai, "Energy Constrained Modulation Optimization," IEEE Trans. Wireless Commun., vol. 4, no. 5, pp. 1-11, 2005.

[6] A. J. Goldsmith and S. B. Wicker, "Design challenges for energy constrained ad hoc wireless networks," IEEE Trans. Wireless Commun., pp. 8-27, 2002.

[7] A. Sahai and P. Grover, "The price of certainty : "waterslide curves" and the gap to capacity," Submitted to IEEE Transactions on Information Theory, Dec. 2007. [Online]. Available: http://arXiv.org/abs/0801.0352v1

[8] P. Massaad, M. Medard, and L. Zheng, "Impact of Processing Energy on the Capacity of Wireless Channels," in International Symposium on Information Theory and its Applications (ISITA), 2004.

[9] S. Verdu, "On channel capacity per unit cost," IEEE Trans. Inform. Theory, vol. 36, pp. 1019-1030, 1990.

[10] P. Massaad, M. Medard, and L. Zheng, "On the capacity of multipleaccess channels with processing power," in Proceedings of the Allerton Conference on Communication, Control, and Computing, Monticello, IL, Oct. 2004.

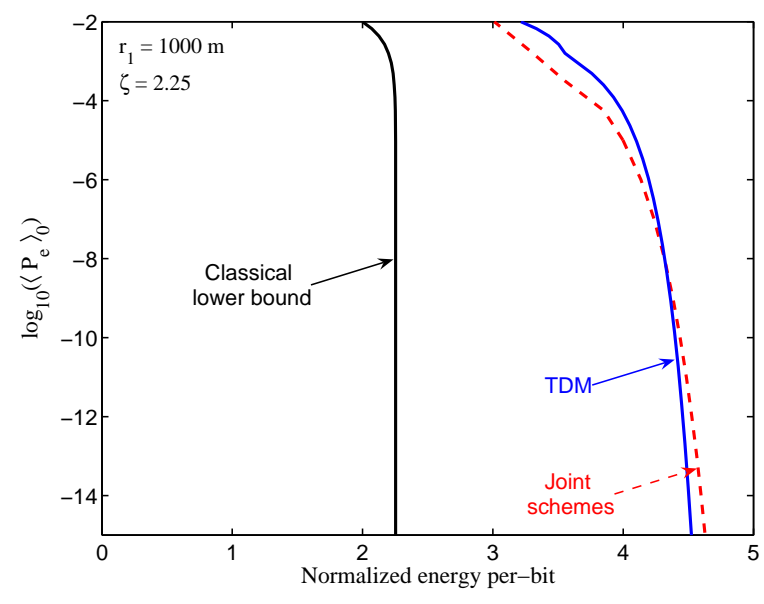

Fig. 3. The plot shows the lower bounds on the (normalized) energy perbit vs $\left\langle P_{e}^{(i)}\right\rangle_{0}$ (equal at the two receivers) for joint strategies and TDM for $\zeta=r_{2}^{2} / r_{1}^{2}=2.25$ at $r_{1}=1000 \mathrm{~m}$. As the error probability decreases, the required decoding energy increases for either scheme. Since TDM saves on decoding energy, it outperforms joint broadcast schemes at low $\left\langle P_{e}^{(i)}\right\rangle_{0}$. The other parameters are the same as that in Fig. 1.

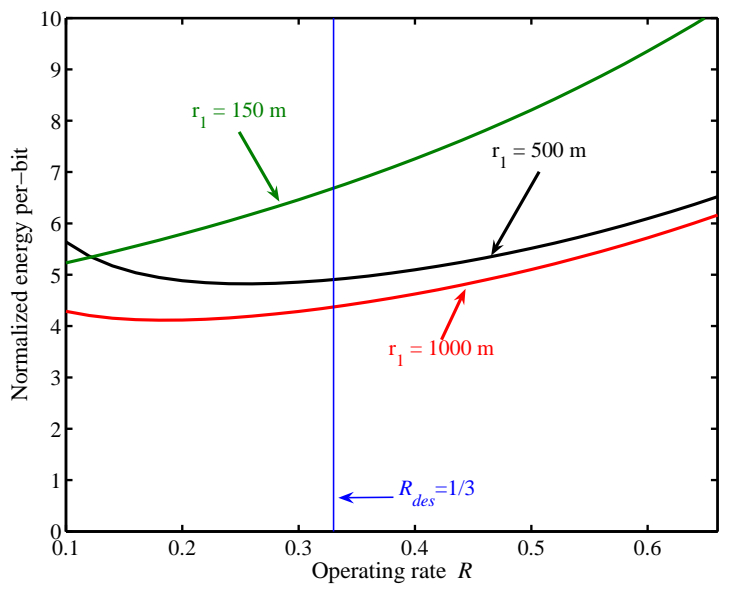

Fig. 4. For joint strategies at moderately large error probabilities $\left(10^{-9}\right.$ in this plot), the optimal communication rate $R^{*}$ that minimizes our lower bounds is 0 at short distances, and close to 0 at large distances. At moderate distances, $R^{*}$ can be large but is observed to be smaller than $R_{\text {des }}=1 / 3$ for the cases considered in this paper.

[11] P. Grover and A. Sahai, "Time-division multiplexing for green broadcasting," Jan. 2009, extended paper. [Online]. Available: http://www.eecs.berkeley.edu/ pulkit/GreenBroadcastingFullProofs.pdf

[12] T. Richardson and R. Urbanke, Modern Coding Theory. Cambridge University Press, 2007.

[13] P. Grover and A. Sahai, "Green codes: Energy-efficient short-range communication," in Proceedings of the 2008 IEEE Symposium on Information Theory, Toronto, Canada, Jul. 2008.

[14] L. Weng, S. S. Pradhan, and A. Anastasopoulos, "Error exponent regions for gaussian broadcast and multiple access channels," IEEE Trans. Inform. Theory, vol. 54, no. 7, pp. 2919-2942, Jul. 2008. 